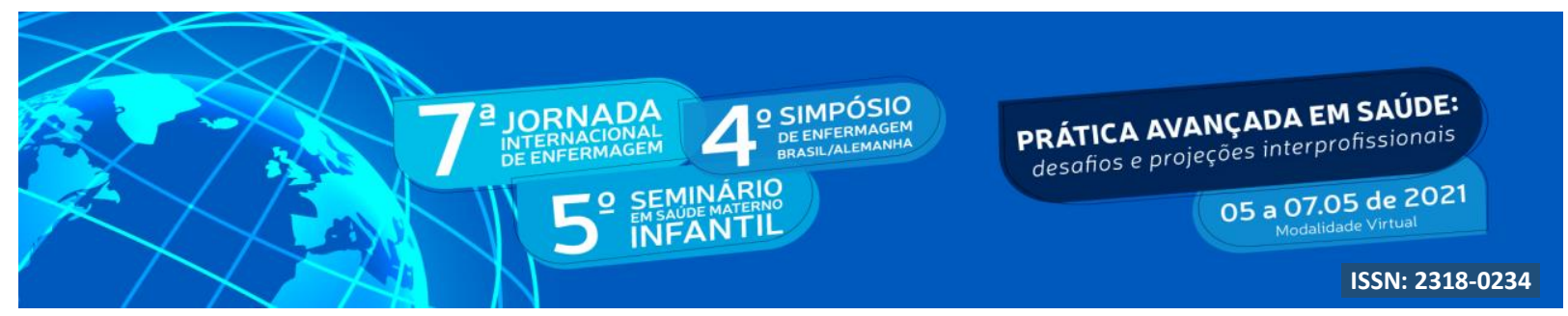

DOI: http://doi.org/10.48195/jie2021-174

\title{
PERCEPÇÕES DE ENTREVISTADORES VOLUNTÁRIOS EM UMA PESQUISA EPIDEMIOLÓGICA SOBRE A COVID-19 NO SUL DO BRASIL
}

\author{
Caroline Bolzan Ilha ${ }^{1}$; Silvana Cruz da Silva; ${ }^{2}$ Edi Franciele Ries ${ }^{3}$; Marinel Mor \\ Dall'agnol $^{4}$
}

\section{RESUMO}

Objetivo: Descrever as percepções de entrevistadores voluntários em uma pesquisa epidemiológica sobre a COVID-19 no sul do Brasil. Método: Estudo descritivo, do tipo relato de experiência. Relato: Para melhor compreensão o relato foi apresentado em dois tópicos: processo de capacitação, onde foi abordado o processo de capacitação e treinamento da equipe de entrevistadores, destacando a realização dos testes rápidos, postura ética na entrevista e utilização dos equipamentos de proteção individual frente a uma pandemia; e a atuação em campo frente a pandemia, a qual discutiu a coleta de dados na prática, sentimentos e percepções dos profissionais como empatia e motivação entre a equipe alguns desafios e estratégias encotradas. Conclusões: Descrever a experiência de entrevistadores voluntários em uma pesquisa de inquérito populacional em meio a pandemia da COVID-19 trás reflexões necessários e importantes para a evolução e reconhecimento da ciência na definição de políticas de enfrentamento.

Palavras-chave: Epidemiologia; Entrevista; Infecções por Coronavirus; Inquéritos Epidemiológicos; Pandemia.

\begin{abstract}
Objective: To describe the perceptions of volunteer interviewers in an epidemiological survey on COVID-19 in southern Brazil. Method: Descriptive study, type of experience report. Report: For better understanding, the report was presented in two topics: qualification process, where the qualification and training process of the team of interviewers was addressed, highlighting the performance of rapid tests, ethical posture in the interview and use of personal protective equipment in front a pandemic; and the performance in the field in the face of the pandemic, which discussed the collection of data in practice, feelings and perceptions of professionals as empathy and motivation among the team, some challenges and strategies found. Conclusions: Describing the experience of volunteer interviewers in a population survey survey in the midst of the COVID-19 pandemic brings necessary and important reflections for the evolution and recognition of science in the definition of coping policies.
\end{abstract}

Key Words: Coronavirus Infections; Epidemiology; Interview; Health Surveys; Pandemics.

\footnotetext{
${ }^{1}$ Enfermeira. Doutoranda da Universidade de Murcia/Espanha. E-mail: carol.ilha@hotmail.com

${ }^{2}$ Enfermeira. Doutora em Enfermagem. Professora da Universidade Franciscana. E-mail: silvanacruzufsm@yahoo.com

${ }^{3}$ Professora do Departamento de Saúde Coletiva, Centro de Ciências da Saúde da Universidade Federal de Santa Maria (UFSM). Email: edi.ries@ufsm.br

${ }^{4}$ Professora associada do Departamento de Saúde Coletiva da Universidade Federal de Santa Maria, RS

(UFSM). Email: marinel@ufsm.br
} 


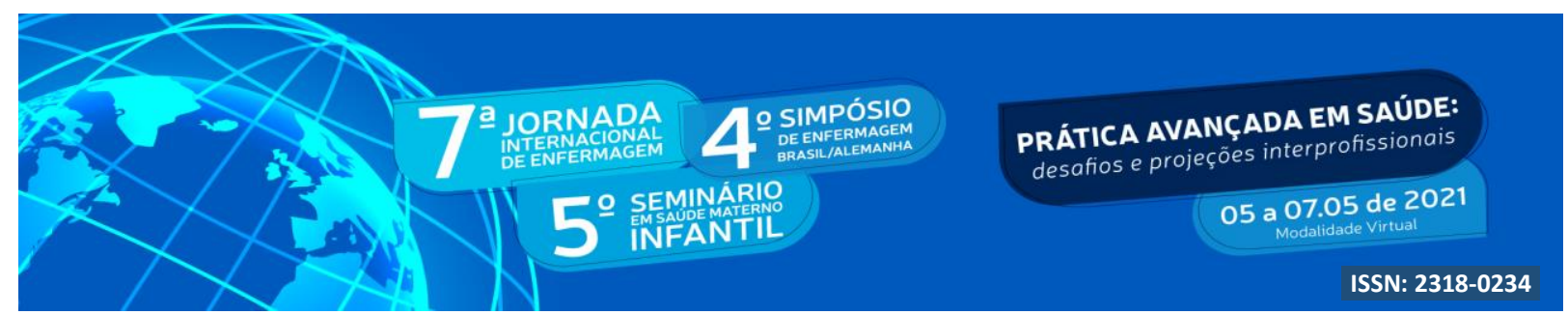

\section{INTRODUÇÃO}

A pandemia pela COVID-19 alterou a vida das pessoas, nos diferentes níveis. Expondoas a constantes modificações sanitárias, educacionais e econômicas (TOUZANI et al., 2020). Desde o seu surgimento em dezembro de 2019, foram confirmados mais de 126 milhões de casos e 2.769.473 milhões mortes até 28 de março de 2021 (WHO, 2021). A taxa de mortalidade associada a doença tem oscilado em aproximadamente 2\% (FAUCI, LANE, REDFIELD, 2020).

Por recomendação da Organização Mundial da Saúde (OMS), diversos países empenham-se em adotar estratégias de enfrentamento embasadas cientificamente e de acordo com a análises de seus contextos. Este fato tem impulsionado pesquisadores do mundo todo no desenvolvimento de estudos. O primeiro levantamento de base populacional foi realizado na Áustria em abril de 2020 com amostras aleatórias de 1.544 austríacos e indicou proporção de 0,33\% de testes positivos (OGRIS; HOFINGER, 2020). Na Islândia, exames foram realizados com amostra aleatória $(\mathrm{N}=2.283)$ e voluntária $(\mathrm{N}=10.797)$ com resultados positivos para $0,6 \%$ e 0,8\% dos testados, respectivamente (GUDBJARTSSON et al., 2020).

O Brasil foi pioneiro no desenvolvimento de uma pesquisa epidemiológica intitulada “Evolução da prevalência de Infecção pela Covid19 (Epicovid-19) no Rio Grande do Sul”, a qual tem dentre os objetivos avaliar a evolução da infecção pelo Coronavírus em nove cidades do Rio Grande do Sul. Coordenada pela Universidade Federal de Pelotas (UFPel) com apoio de doze universidades gaúchas e do governo estadual para subsidiar a tomada de decisões de enfrentamento neste contexto (UNIVERSIDADE FEDERAL DE PELOTAS, 2020). Para viabilizar o desenvolvimento desta pesquisa, muitas foram as instituições, pesquisadores e colaboradores envolvidos desde sua elaboração até as fases de coleta, análise e divulgação dos resultados.

Neste contexto pandemico, todas as informações e evidências compartilhadas, são importantes para o desenvolvimento de estratégias de enfrentamento a COVID-19. Assim, com esse relato busca-se divulgar a experiência de entrevistadores voluntários acerca do processo estrutural, capacitações, organização e planejamento da participação em uma pesquisa de 


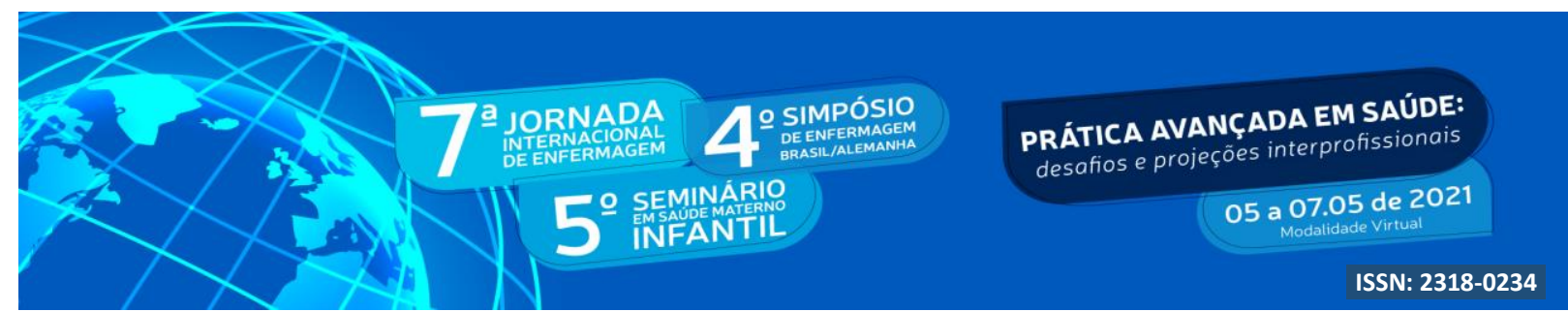

inquérito populacional, bem como das questões subjetivas dos colaboradores, as motivações, facilidades e desafios.

\section{OBJETIVO}

Frente ao exposto, tem-se como objetivo descrever as percepções de entrevistadores voluntários em uma pesquisa epidemiológica sobre a COVID-19 no sul do Brasil.

\section{METODOLOGIA}

Trata-se de um estudo descritivo, do tipo relato de experiência, sobre o treinamento (capacitações) e vivencias de voluntários que atuaram como entrevistadores e apoiadores (colaboradores) para a produção de dados de uma pesquisa de base populacional sobre a COVID -19, na cidade brasileira de Santa Maria, Rio Grande do sul Brasil.

A pesquisa objeto da vivência relatada é uma iniciativa da Secretaria de Saúde, da Vigilância Epidemiológica e do Governo do Estado do Rio Grande do Sul, encomendada à UFPel e executada em parceria com outras Universidades gaúchas. Configurada, a priori, com quatro inquéritos iniciados em março de 2020, já realizou-se 9 inquéritos e segue em andamento em nove municípios: Pelotas, Santa Maria, Uruguaiana, Ijuí, Passo Fundo, Caxias do Sul, Santa Cruz do Sul, Porto Alegre e Canoas (HALLAL, et al. 2020).

Em cada município, foram sorteados aleatoriamente os domicílios que entram no estudo; e em cada domicílio, um novo sorteio determinava o morador que iria realizar o teste rápido. A experiência relatada é referente ao período de participação nas coletas de abril a agosto de 2020, na cidade de Santa Maria-RS, o que correspondeu a seis inquéritos.

Os registros discutidos referem-se as vivências de três voluntárias: uma enfermeira, pósdoutoranda em enferamgem e docente da Universidade Franciscana (entrevistadora), uma enfermeira doutoranda da Universidade de Murcia- Espanha (entrevistadora) e uma farmacêutica e docente em Saúde Coletiva (entrevistadora e apoio de campo).

Para melhor compreensão este relato será apresentado em dois tópicos: processo de capacitação e a atuação em campo frente a uma pandemia. 


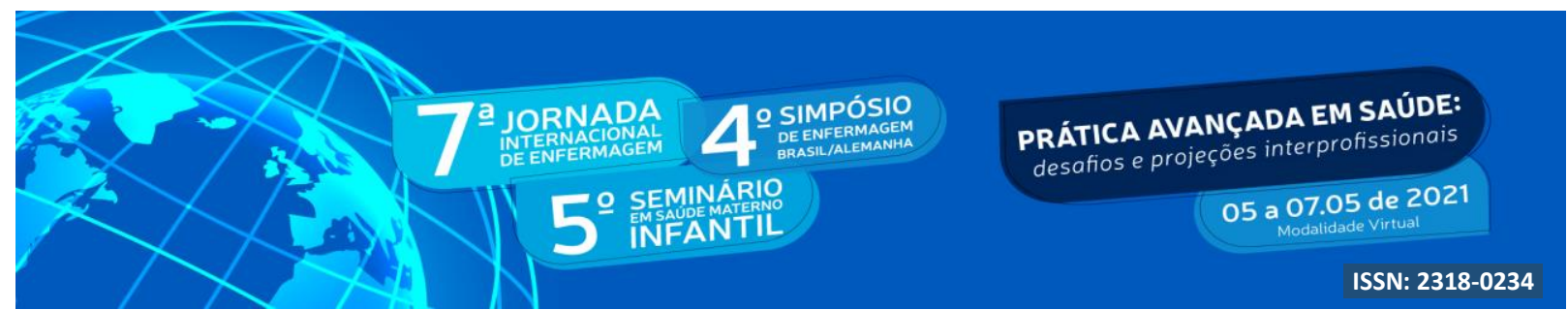

\section{RESULTADOS E DISCUSSÕES}

\section{Processo de capacitação}

Previamente a realização do trabalho de campo a cada inquérito, os participantes voluntários realizavam inscrições e eram selecionados via análise curricular, por um Núcleo de Coordenação local composto por professores da Universidade Federal de Santa Maria.

Os voluntários realizavam o treinamentos de Requalificação e testagem prévia por meio dos testes rápidos para Covid-19, sob supervisão do Instituto Pesquisas de Opinião (IPO), disponibilizados pelo Ministério da Saúde (MS). Os treinamentos tinham duração de aproximadamentes seis horas, nos quais além da abordagem de medidas de biossegurança, protocolos de testagem, conduta diante de casos positivos, da técnica de seleção de amostragem dos domicílios, dos entrevistados testados (indivíduos maiores de 18 anos e de ambos os sexos) e do preenchimento da documentação, eram compartilhadas estratégias para evitar/solucionar problemas comuns em pesquisa de campo.

Dessa forma, a cada inquérito, eram realizadas essas capacitações e testagem dos entrevistadores e da equipe de apoio, na sexta-feira e na sequência, iniciava-se o trabalho de campo até a sua conclusão no domingo. Seguem imagens, na Figura 1, de algumas orientações realizadas.

Figura 1- Realização e resultado e dos testes rápidos.

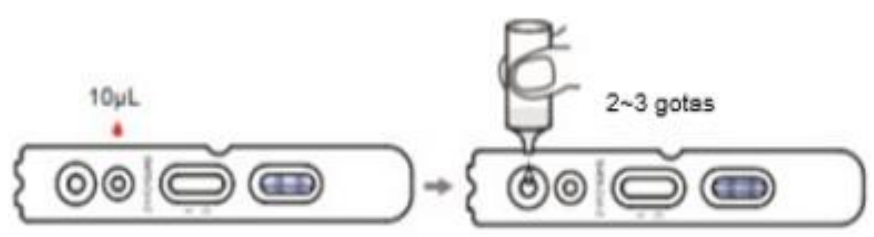

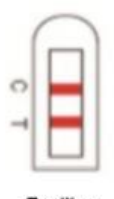

Positivo

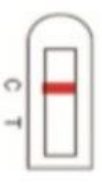

Negativo

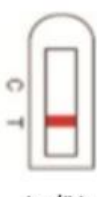

Inválido

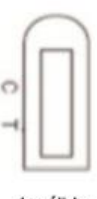

Inválido

Fonte: Imagem adaptada da bula do teste.

A ampliação dos questionários digitais, foi um dos pontos bastante discutidos e referemse a entrevista propriamente dita, tendo em vista o número de 69 questões, as quais eram realizadas nos celulares dos entrevistadores. Assim, cada questão e suas opções foram esclarecidas nos treinamentos. A necessidade de obtenção de respostas verossímeis em uma entrevista, destaca a relevância de uma postura ética e profissional por parte do entrevistador, para não criar relações de indução ou autoridade com o entrevistado (MINUZZI, et. al., 2019). 


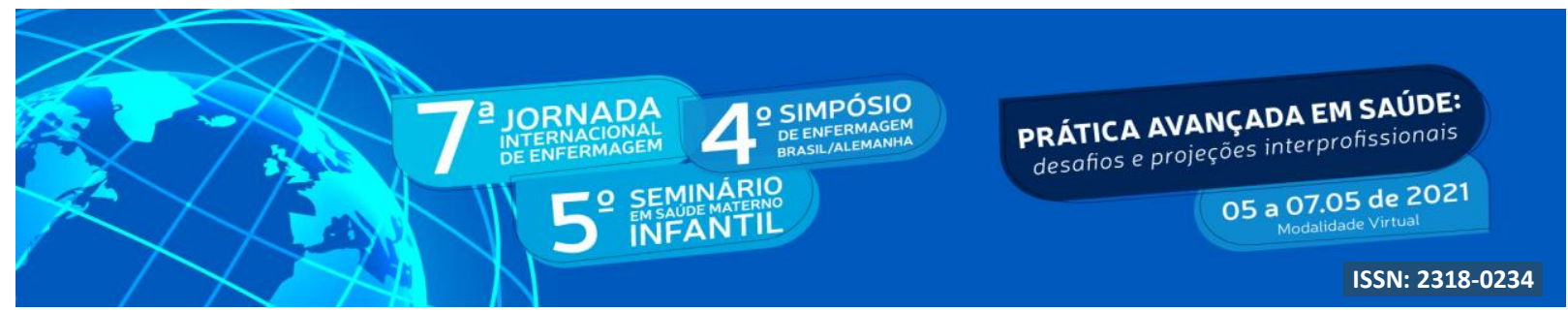

Nas capacitações, um ponto essencial foi a implementação e adesão a protocolos rígidos de biossegurança, sendo ressaltada a importância do auto-cuidado, da higienização das mãos e dos materiais utilizados, principalmente ao entrar nos domiciolios das pessoas. Além disso, da utilização dos equipamentos de proteção individuais (EPIS), entende-se que investir no conhecimento, capacitação e treinamento dos profissionais sobre o uso adequado desses equipamentos, da paramentação a desparamentação, é uma forma eficaz de se evitar a contaminação pelo coronavírus (OLIVEIRA, SOUZA, LEITE, CAMPOS, 2020).

Esta forma de organização vai ao encontro dos princípios de gerenciamento de emergências preconizados pela OMS (OMS, 2020) e do Plano de Contingência Nacional para Infecção Humana pelo novo Coronavírus COVID-19 (MS, 2020), no que se refere as capacidades e sistemas de respostas rápidas e eficazes em casos de desastres em emergência em saúde necessários para o enfrentamento da pandemia. Tendo em vista que são poucos os estudos sobre o tema, os protocolos e recomendações sofrem mudanças constantes (RODRIGUES, SILVA, 2020).

Além disso, a COVID-19 é uma enfermidade infecciosa transmitida, principalmente, pelo contato e por gotículas respiratórias provenientes de pacientes sintomáticos (MS, 2020) e assintomáticos, ou seja, sem sinais e sintomas que estejam infectados (NASCIMENTO et al., 2020). Por se tratar de uma doença recente, em seres humanos, existem lacunas de evidências científicas quanto ao percurso e tratamento da doença, as quais estão sendo investigadas por pesquisadores em todo o mundo (ANDERSEN et al., 2020), o que reforça a importância de capacitações voltadas aos profissionais de diferentes frentes.

\section{Atuação em campo frente a pandemia}

Conforme as orientações recebidas na capacitação, iniciou-se a fase de coletas de dados com deslocamento aos bairros sorteados. Cada dupla em seu veículo automotivo identificado, para facilitar o reconhecimento da população e, também, a logística de acesso em vias públicas.

O processo logístico envolvendo o deslocamento de carro e a pé até o endereço sorteado, o processo de aleatorização necessários metodologicamente no estudo e abordagem dos participantes foram desafiadores, principalmente porque a OMS enfatiza que que não existe uma abordagem única para o gerenciamento de casos e que os países devem adotar uma mistura de intervenções com base em uma análise da situação e do contexto local (WHO, 2020c). 


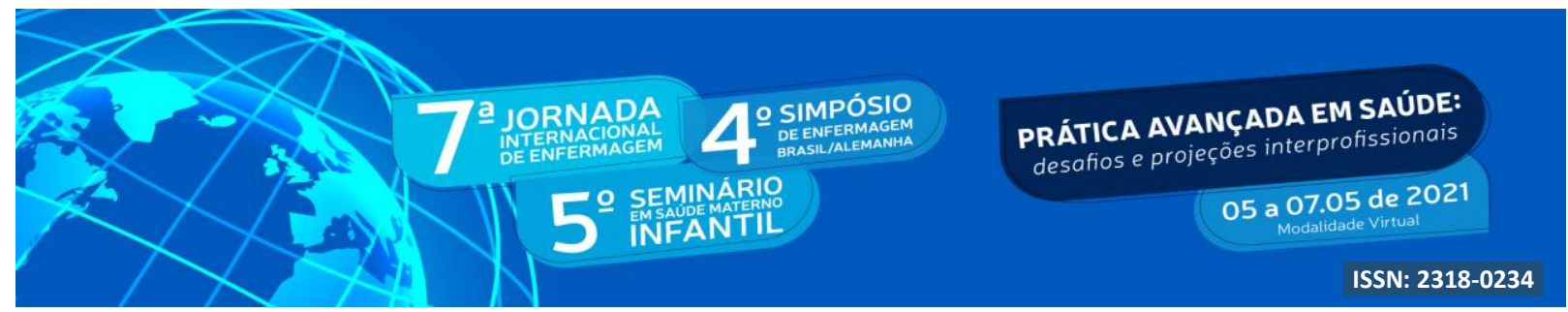

Contudo, a formação incialmente em duplas, constituída por um entrevistador e um apoio, foi fundamental para acolher expectativas que permeavam o momento delicado o qual vivenciava-se como profissionais da saúde voluntários. Sendo essencial para que se obtivesse segurança no desempenho das atribuições. A dinâmica de trabalho em equipe é fundamental para o compartilhamento de sentimentos de coragem e resignação que impulsionava os participantes frente ao desconhecido, promovendo a ação necessária para cumprimento do dever ético, profissional e moral. Ao mesmo tempo que amparava receio e medo diante das primeiras abordagens em campo (MINUZZI, et. al., 2019).

Conjuntamente, contou-se com o apoio de uma equipe de retaguarda, formada pelos coordenadores locais da pesquisa e supervisores do IPO, para resolução de problemas ou dúvidas que surgissem no decorrer do dia. Essa equipe prestava remotamente auxílio logístico, por meio de grupo em WhatsApp e contato telefônico fornecendo orientações e, também, acionando reforços como a Guarda Municipal em situações que houvesse necessidade por risco de segurança aos voluntários.

Ao avançar das coletas e dos inquéritos, verificou-se o surgimento de uma nova e importante rede de apoio entre os próprios voluntários compartilhando como estava sendo a experiencia em tempo real pelo grupo de WhatsApp. Nessas trocas, eram emitidos alertas sobre potenciais pontos de dificuldades, bem como estratégias que poderiam auxiliar o trabalho de todos. A exemplo, cita-se a dificuldade de contatar um participante sorteado quando esse era morador de um prédio, seja por problemas com interfone, receio de permitir a entrada de um estranho no prédio ou mesmo pelo desafio de sensibilizar o indivíduo sobre a importância da sua participação quando não está ocorrendo um contato visual entre os interlocutores.

Logo que as primeiras situações surgiram, os voluntários compartilharam estratégias no grupo, como ofertar ao participante a alternativa de se deslocar até o entrevistador para conversar antes de permitir sua entrada no prédio, realizar o teste no carro do entrevistador ou ainda acionar o síndico e solicitar a mediação da comunicação. No entanto, cabe ressaltar situações nas quais a entrada nos prédios foi impossibilitada pelo síndico, e assim, outras alternativas eram utilizadas como solicitar contato desse com coordenadores e supervisores da pesquisa, ou ainda, a chamada da Guarda Municipal para respaldar a veracidade da visita dos entrevistadores. 


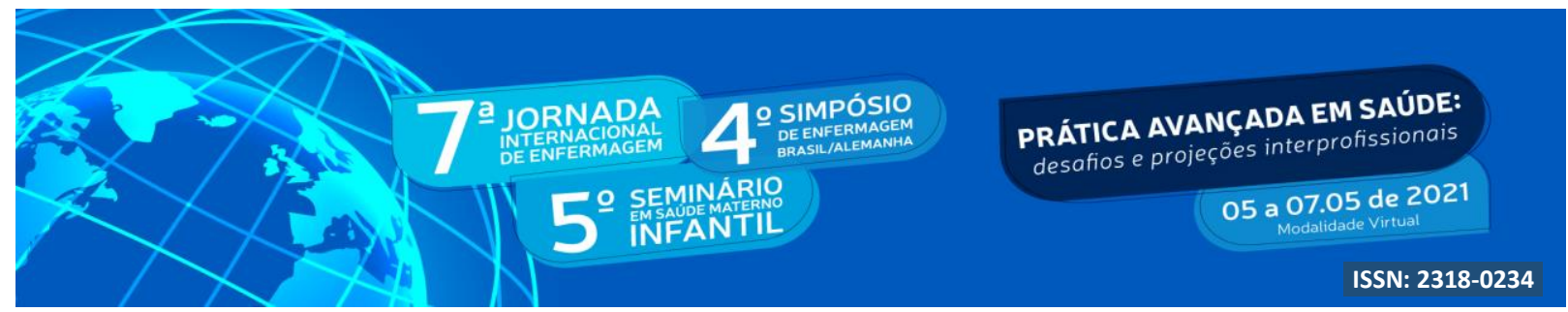

Ainda se tratando de moradores sorteados em prédios, destaca-se o grande número de abordagens não efetivadas, seja pela recusa de participação pelos motivos supracitados como receio por segurança e/ou desafio de sensibilização à distância, ou por não atendimento. Em alguns locais, vizinhos informavam a ausência dos moradores em virtude de horário de trabalho ou viagem. Em outros, considerando regiões habitadas tipicamente por estudantes, acredita-se que a evasão decorrente da situação pandêmica onde muitos alunos retornaram a suas cidades natais para seguir os estudos remotamente pode ter sido um fator contribuinte para a ausência de atendimentos.

O registro minucioso das abordagens não efetivadas é tão importante quanto aquelas realizadas para a validade da pesquisa epidemiológica e requiriu um importante tempo do trabalho de campo. Cita-se uma situação com entrevistadora no terceiro inquérito que teve índice superior a $85 \%$ de não atendimentos e/ou recusas. Em tais casos destaca-se o papel fundamental desempenhado pelo apoio de campo, fornecendo o suporte necessário para agilizar o processo de registro e identificação de próxima abordagem para a sequência do trabalho do entrevistador.

Outro desafio à fluidez das abordagens efetivas decorria da incompatibilidade de mapas de alguns setores censitários com os locais encontrados. Muitas regiões ampliaram-se nos últimos anos e dessa forma, alguns mapas - norteadores para os processos de amostragem apresentavam divergências em nomes de ruas ou mesmo surgimento de áreas habitadas em regiões não identificadas previamente. Todo processo de compreensão e encaminhamento junto ao núcleo coordenador envolvia tempo e era facilitado quando o entrevistador estava acompanhado de um apoio de campo. Esse, além de realizar a comunicação com o núcleo coordenador definindo a resolução do problema enquanto o entrevistador efetivava uma abordagem, atuava na análise de mapas para o diagnóstico prévio de tais situações.

Nesse contexto é importante compreender como a resiliência se aplicou no processo de trabalho dos sujeitos envolvidos em uma pesquisa de campo no momento de uma pandemia. Avaliar a resiliência no trabalho constitui um importante meio de autoconhecimento para os trabalhadores, assim como subsídios para as organizações (GRECO, 2018).

Os desafios foram sendo amenizados com a maior divulgação da pesquisa nos meios de divulgação e conforme o decorrer da realização dos inquéritos. Assim a amostragem sorteada foi apresentado melhor receptividade e gratidão pela participação no estudo. Muitos indivíduos 


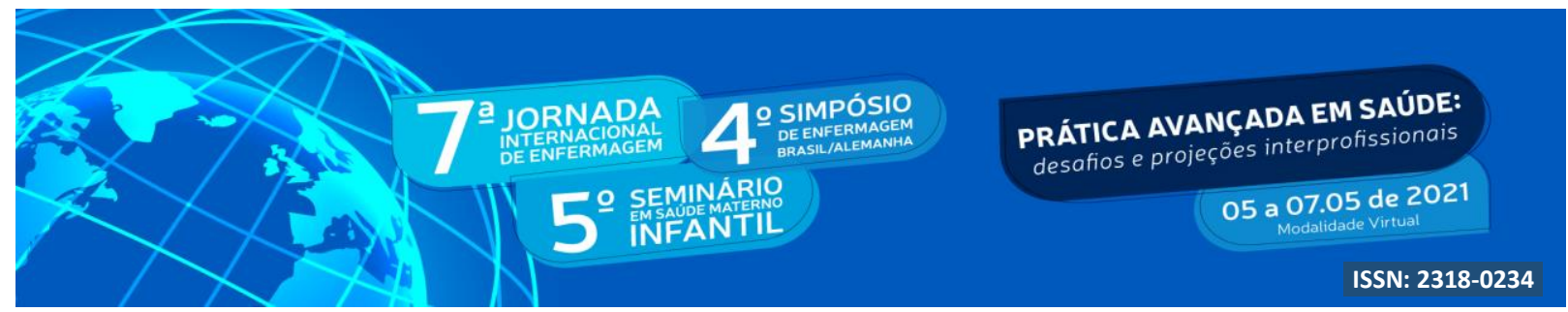

demonstravam reconhecimento ao trabalho voluntário realizado, solicitavam permissão para registrar o momento junto à entrevistadora e eram incansáveis nos agradecimentos pela oportunidade de serem testados para COVID-19. Em alguns casos, as pessoas ofertavam gentilezas como cadeiras, comidas e bebidas, mas compreendiam que o rigor metodológico da pesquisa não possibilitava o aceite e novamente retribuíam com palavras de gratidão.

Vivenciou-se também, sentimentos de gratidão por participantes que estavam há muito tempo em isolamento, cumprindo com a recomendação de distanciamento social entre familiares e amigos, tendo com a participação na pesquisa uma oportunidade de diálogo. Relatos de solidão e impactos psicossociais negativos da pandemia foram registrados por participantes de diferentes faixas etárias, mas em maior grau, pela população idosa. Esses casos oportunizaram as entrevistadoras um momento de maior empatia, escuta ativa e reforço sobre a importância dessa medida de distanciamento para a contenção da pandemia. Ao final da entrevista, esses participantes de alguma forma também tinham seu esforço reconhecido e sentiam-se gratos por colaborarem, dados semelhantes são encontrados em relatos sobre outras participações em pesquisas (MINUZZI, et. al.; 2019).

O momento da entrevista também foi uma oportunidade para sanar dúvidas. Alguns participantes questionavam sobre aspectos bem pontuais da pesquisa como a sensibilidade e qualidade do teste utilizado, método de aleatorização de domicílios ou sorteio do morador que iria ser testado. Outros tinham dúvidas relacionadas à origem do vírus, sobre fake News nas redes sociais, remédios caseiros ou farmacoterapias preventivas. Todas foram oportunidades de diálogo e esclarecimentos ímpares na promoção de impacto e transformação social pelo compartilhamento de conhecimentos científicos, uma vez que a prevenção é a principal medida para combater a COVID-19 (BRASIL, 2020).

\section{CONCLUSÃO}

A partir desse relato foi possivel conhecer as percepções e experiencias de entrevistadores voluntários em uma pesquisa epidemiológica sobre a COVID-19. Reflete-se que frente a um momento tão delicado de pandemia, é fundamental a valorização dos profissionais que buscam estratégias para ampliar o que se conhece sobre o vírus. Da mesma forma alguns sentimentos e aspectos foram fortalecidos como a resiliência, a gratidão e o reconhecimento. 


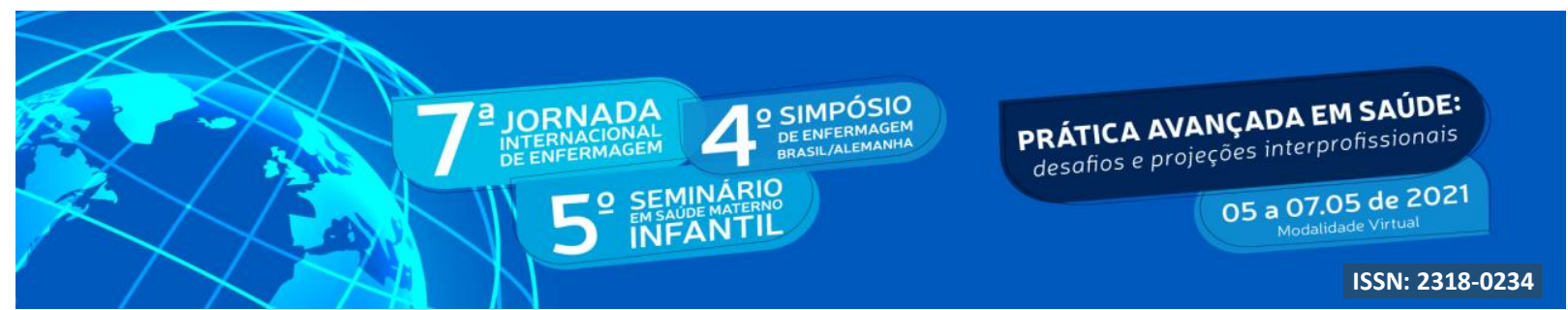

Outro fator importante é o adequado planejamento das pesquisas epidemiologicas que dependem do envolvimento de muitos seres-humanos para sua efetivação, bem como do apoio e de um bom trabalho em equipe para que os resultados sejam condizentes e fidedignos ao método.

As informações e evidências compartilhadas, os desafios e as facilidades no desenvolvimento de estratégias de enfrentamento a COVID-19 são reflexões necessárias e importantes para a evolução e reconhecimento da ciência na definição de políticas de enfrentamento.

\section{REFERÊNCIAS}

ANDERSEN, K.G. et al. The proximal origin of SARS-CoV-2. Nature Medicine, 26; 450455. 2020. doi:10.1038/s41591-020-0820-9

BRASIL. LEI 7.498, De 25 de Junho de 1986. Dispõe sobre a regulamentação do exercício da enfermagem e dá outras providências. BRASÍLIA: Ministério Da Saúde; 1986. [Citado em 05 Maio 2008]. Disponível em: http://www2.camara.gov.br/internet/legislacao/legin.htm.

BRASIL. Ministério da Saúde. Agência Nacional de Vigilância Sanitária -ANVISA. Nota Técnica No 04/2020. Orientações para serviços de saúde: medidas de prevenção e controle que devem ser adotadas durante a assistência aos casos suspeitos ou confirmados de infecção pelo novo coronavírus (covid-19).

FAUCI, A.S.; LANE, H.C.; REDFIELD, R.R. Covid-19 - Navigating the Uncharted [published online ahead of print, $2020 \mathrm{Feb} 28$ ]. N Engl J Med. 2020;10.1056/NEJMe2002387. https://doi.org/10.1056/ NEJMe2002387

FLICK, U. Introdução á pesquisa qualitativa. Porto Alegre: Artmed, 2009.

GRECO, P.B.T. Adaptação transcultural para língua portuguesa do Brasil da Resilience at Work Scale (RAW Scale)/Patricia Bitencourt Toscani Greco. - 2018. 230 p.

GUDBJARTSSON, D.F. et al. Spread of SARS-CoV-2 in the Icelandic population. N Engl J Med Apr 14; [e-pub]. 2020.

HANASHIRO,D. M. M. ET AL. Gestão do Fator Humano: uma visão baseada em stakeholders. São Paulo: Saraiva, 2007.

HALLAL PC et al. Trends in the prevalence of COVID-19 infection in Rio Grande do Sul, Brazil: repeated serological surveys. Ciência \& Saúde Coletiva, 25(Supl.1):2395-2401, 2020 


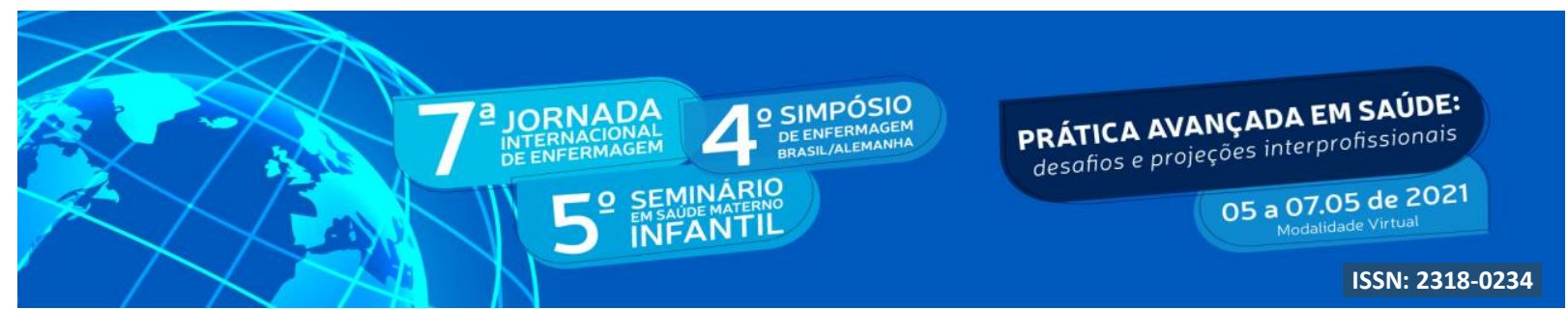

MINUZZI, NATÁLIA M.; VILANOVA, KAMILA D.; MAINARDI, GABRIELLA O.; RIES, EDI F.; A importância da capacitação do pesquisador-entrevistador em pesquisas avaliativas de serviços públicos de saúde. Revista Saúde (Sta. Maria). 2019; 45 (3)

NASCIMENTO, I J B; et al, 2020. Novel Coronavirus Infection (COVID-19) in Humans: A Scoping Review and Meta-Analysis. J. Clin. Med. 2020, 9(4), 941; https://doi.org/10.3390/jcm9040941

OLIVEIRA HC, SOUZA LC, LEITE TC, CAMPOS JF. Equipamento de Proteção Individual na pandemia por coronavírus: treinamento com Prática Deliberada em Ciclos Rápidos. Rev Bras Enferm. 2020;73(Suppl 2): http://dx.doi.org/10.1590/0034-7167-2020-0303 e20200303

OGRIS, G.; HOFINGER, C. “COVID-19 Prevalence (Media information).” Disponível em: https://www.sora.at/uploads/media/Austria_COVID-

19_Prevalence_BMBWF_SORA_20200410_EN_Version_fuer_HP.pdf. Acesso em $01 \mathrm{fev}$ 2021.

UNIVERSIDADE FEDERAL DE PELOTAS - UFPel. 2020. Covid-19: UFPel coordena estudo populacional sobre a pandemia no RS. Disponível em http://www.epidemioufpel.org.br/site/content/sala_imprensa/noticia_detalhe.php?noticia=3092. Acesso em 28 mar 2021.

RODRIGUES NH, SILVA LGA. Gestão da pandemia Coronavírus em um hospital: relato de experiência profissional. J. nurs. health. 2020;10(n.esp.):e20104004 https://periodicos.ufpel.edu.br/ojs2/index.php/enfermagem/article/view/18530/11238

TOUZANI, R. et al. Coronavirus, Covid19, Covid-19 and SARS-Cov-2: A Global Pandemic, A Short Review. J. Mater. Environ. Sci., 11(5): 736-750, 2020.

WHO. Coronavirus disease (COVID-19) pandemic. Numbers at a glance. Disponível em: https://www.who.int/emergencies/diseases/novel-coronavirus-

2019?gclid=Cj0KCQjw0oCDBhCPARIsAII3C_EzlOTzWPbYV5LsuLoA8F1N1LjI0vtckm31 hw8rxpCm9TemvWN9xDQaAi2dEALw_wcB. Acesso em 28 mar 2021.

WORLD HEALTH ORGANIZATION (WHO). Coronavirus disease 2019 (COVID-19) Situation Report 56. Geneve: WHO [Internet]. 2020 [cited 2021 Mar 19]. Available from: https://www.who.int/docs/default-source/coronaviruse/situation-reports/20200311- sitrep-51covid-19.pdf

WHO. WHO announces COVID-19 outbreak a pandemic. 2020c. Disponível em: : http://www.euro.who.int/en/health-topics/health-emergencies/coronavirus-covid19/news/news/2020/3/who-announces-covid-19-outbreak-a-pandemic. Acesso em 02 mai 2020. 